\title{
Fecal Microbiota Transplant from a Rational Stool Donor Improves Hepatic Encephalopathy: A Randomized Clinical Trial
}

\author{
Jasmohan S. Bajaj, MD' ${ }^{1}$, Zain Kassam, MD ${ }^{2,5}$, Andrew Fagan, BS ${ }^{1}$, Edith A. Gavis, RN ${ }^{1}$, \\ Eric Liu ${ }^{3}$, I. Jane Cox, PhD ${ }^{4}$, Raffi Kheradman ${ }^{3}$, Douglas Heuman, MD ${ }^{1}$, Jessica Wang ${ }^{3}$, \\ Thomas Gurry, $\mathrm{PhD}^{5}$, Roger Williams, MD ${ }^{4}$, Masoumeh Sikaroodi, $\mathrm{PhD}^{3}$, Michael Fuchs, \\ MD ${ }^{1}$, Eric Alm, $\mathbf{P h D}^{5}$, Binu John, $\mathbf{M D}^{1}$, Leroy R Thacker, $\mathrm{PhD}^{1}$, Antonio Riva, $\mathrm{PhD}^{4}$, Mark \\ Smith, PhD $^{2}$, Simon D. Taylor-Robinson, MD $^{6}$, and Patrick M Gillevet, PhD $^{3}$ \\ ${ }^{1}$ Virginia Commonwealth University and McGuire VA Medical Center, Richmond, Virginia, USA \\ 2OpenBiome, Somerville, Massachusetts, USA \\ ${ }^{3}$ George Mason University, Manassas, Virginia, USA \\ ${ }^{4}$ Institute of Hepatology London, Foundation for Liver Research, United Kingdom \\ ${ }^{5}$ Massachusetts Institute of Technology, Cambridge, Massachusetts, USA \\ ${ }^{6}$ Imperial College, London, United Kingdom
}

\section{Abstract}

Recurrent hepatic encephalopathy (HE) is a leading cause of readmission despite standard of care (SOC) associated with microbial dysbiosis. Fecal microbiota transplantation (FMT) may improve dysbiosis; however, it has not been studied in HE. We aimed to define whether FMT using a rationally-derived stool donor is safe in recurrent HE compared to SOC alone. An open-label, randomized clinical trial with a 5 month follow-up in outpatient cirrhotic men with recurrent HE on SOC was conducted with 1:1 randomization. FMT-randomized patients received 5-days of broad-spectrum antibiotic pre-treatment then a single FMT enema from the same donor with the optimal microbiota deficient in HE. Follow-up occurred on days 5, 6, 12, 35 and 150 postrandomization. The primary outcome was safety of FMT compared to SOC using FMT-related serious adverse events (SAE). Secondary outcomes were AEs, cognition, microbiota and metabolomic changes. Participants in both arms were similar on all baseline criteria and were followed till study-end. FMT with antibiotic pre-treatment was well-tolerated. Eight (80\%) SOC participants had a total of 11 SAE compared to two (20\%) FMT participants with SAEs (both FMT-unrelated, $\mathrm{p}=0.02$ ). Five SOC and no FMT participants developed further HE ( $\mathrm{p}=0.03)$. Cognition improved in FMT, but not SOC group. MELD score transiently worsened postantibiotics, but reverted to baseline post-FMT. Post-antibiotics, beneficial taxa and microbial

Corresponding Author: Jasmohan S Bajaj, MD, Division of Gastroenterology, Hepatology and Nutrition, Virginia Commonwealth University and McGuire VA Medical Center, 1201 Broad Rock Boulevard, Richmond, VA 23221, jasmohan.bajaj@vcuhealth.org, Telephone: (804) 675 5802, Fax: (804) 6755816.

Author contributions: JSB conceptualized the trial with input from ZK and DMH and was involved in all aspects, ZK, MS, EA and TG were involved in donor selection procedures, PMG, MS, EL, RK, JW were involved in microbiota and bioinformatics analyses, LRT was the study biostatistician, AF, EAG, DMH, BJ and MF were involved in patient recruitment and follow-up, SDT, IJC, RW, AR were involved in metabolomic analyses and all authors were involved in drafting and critical revision of the manuscript. 
diversity reduction occurred with Proteobacteria expansion. However, FMT increased diversity and beneficial taxa. SOC microbiota and MELD score remained similar throughout.

Conclusions: FMT from a rationally selected donor reduced hospitalizations, improved cognition and dysbiosis in cirrhosis with recurrent HE.

\section{Keywords}

Cirrhosis; Dysbiosis; Hospitalizations; Metabolomics; Stroop App

Cirrhosis is a leading cause of morbidity and mortality, most rooted in liver-associated complications such as hepatic encephalopathy $(\mathrm{HE})(1),(2,3)$. Many of these patients are exposed to antibiotics, which disrupts gut microbiota, precipitating further $\operatorname{HE}(4,5)$. Standard of care (SOC) treatments such as lactulose and rifaximin can change the gut microbial functionality and milieu, however, they are often ineffective and recurrent HE can result in cumulative, irreversible brain injury(6-8). HE patients have a reduced relative abundance of beneficial short-chain fatty acid (SCFA) producing families such as Lachnospiraceae and Ruminococcaceae, and enrichment of potentially pathogenic Enterobacteriaceae(9-13). This microbial profile has been linked to cognitive impairment and systemic inflammation which can predict negative outcomes $(14,15)$. Accordingly, microbiome-directed therapy that can complement microbial deficiencies may reduce HE recurrence.

Fecal microbiota transplants (FMT) have been shown in randomized-controlled trials to be effective in dysbiotic conditions such as recurrent Clostridium difficile and ulcerative colitis(16-19). Cross-sectional data demonstrating microbial dysbiosis in $\mathrm{HE}(20)$ and a case report(21) suggest FMT may be promising. However, given data suggesting heterogeneity in stool donor efficacy in ulcerative colitis(18), we chose to use a single rationally identified donor for complementing the unique deficiencies among HE patients. The aim of our openlabel, randomized clinical trial was to assess the safety, impact on liver and cognitive function of patients undergoing FMT using a rationally-derived donor in recurrent HE.

\section{EXPERIMENTAL PROCEDURES}

\section{Rational Donor Selection}

Using microbiome data from HE and healthy control participants, we performed a machine learning technique to identify a single donor who had the highest relative abundances of Lachnospiraceae and Ruminococcaceae among a universal stool donor bank (Supplementary Information). All donor material came from a single stool specimen provided by this donor for all FMT-assigned patients.

\section{Study Design}

This was an open-label, randomized clinical trial among cirrhotic patients with recurrent HE on SOC comparing FMT preceded by antibiotic pre-treatment (FMT arm) versus SOC alone (SOC arm). This trial was carried out under FDA IND 16542 at the McGuire VAMC and approved by the Institutional Review Board. The trial is registered at www.clinicaltrials.gov (NCT02636647). All participants provided informed consent and an independent data safety 
monitoring board (DSMB) evaluated the trial on an ongoing basis. Participants underwent screening procedures including complete blood count, model for end-stage liver disease (MELD) calculation(22), stool analysis for $C$. difficile and a 24-hour dietary recall. If eligible, participants were randomized 1:1 using a random number generator into FMT or SOC arm. Participants were evaluated at days $0,5,6,12,20$ and 35 and 150 postrandomization for a safety assessment (Figure 1A). All subjects were instructed to maintain their regular Western diet during the trial, which was confirmed at each visit.

\section{Study Population}

Twenty eligible patients were $\geq 18$ years cirrhotic outpatients with recurrent HE defined as at least two documented overt HE episodes requiring therapy, and supervised by an adult caregiver. Patients were excluded if they had a MELD score $>17$, on oral or intravenous antimicrobial agents besides non-absorbable rifaximin, allergies to pre-treatment antibiotics, immunosuppressive medications, positive $C$. difficile test, pregnancy, active infection, those with active alcohol abuse and unable to provide informed consent (supplementary Table1).

\section{Baseline Assessment}

Potential participants were evaluated for demographic information, cirrhosis/HE history, medication profile, and underwent two validated neuro-cognitive tests, psychometric hepatic encephalopathy score (PHES) and EncephalApp-Stroop(1,23). PHES is a five-test battery which yields a composite standard deviation score compared to local norms while the time required to complete five runs in the On and Off state by EncephalApp-Stroop (OffTime +OnTime) is recorded (Supplementary information)(23). Blood samples were drawn for hepatic panel and complete blood count. Stool was analyzed at baseline to rule out $C$. difficile and other enteric infections. A separate stool aliquot was collected in RNALater for 16s rRNA microbiome analysis(10). Urine samples during the visit were snap-frozen for NMR metabolomics(24).

\section{Intervention}

Participants randomized to the FMT arm were given pre-treatment antibiotics to decrease the host bacterial load, lower diversity and enable a colonic colonization of the donor microbiota(25). Lactulose and rifaximin were continued for all patients throughout the trial. A five-day broad spectrum coverage regimen was used (metronidazole $500 \mathrm{mg}$ orally three times daily, ciprofloxacin $500 \mathrm{mg}$ orally twice daily and amoxicillin 500mg orally three times daily). All antibiotics were discontinued at least 12 hours before FMT to ensure these agents would not impact the instilled microbiota. FMT was prepared from a healthy volunteer from a universal stool bank (OpenBiome) (Supplementary Information). Three frozen-then-thawed FMT units ( $90 \mathrm{~mL}$ total) were instilled by enema and retained for 30 minutes(26). Participants in the SOC arm did not receive antibiotic pre-treatment or FMT but follow-up was identical on days 5, 6, 12, 35 and 150 post-randomization (Figure 1A)

\section{Clinical Outcomes}

The primary outcome was proportion of participants with FMT-related serious adverse events (SAEs) at day 150, a composite endpoint of death, hospitalizations, ER visits or 
transmissible infections, as defined by the FDA. The secondary outcomes were changes in cognitive function at day 20, cirrhosis severity (MELD score, albumin), changes in liver function and WBC count, development of all AEs, changes in microbiota composition and function in the FMT arm compared to SOC arm. In addition to SAE, solicited AEs events at each visit included: 1) gastrointestinal symptoms (diarrhea, abdominal pain, bloating); 2) liver-associated (HE, jaundice, changes in liver function); 3) infections; 4) related to enema (rectal bleeding/trauma).

\section{Assessment and Analysis of Microbiome and Metabolomics}

Stool was analyzed for microbiota composition using 16s rRNA sequencing using Multitag sequencing on an Ion Torrent PGM(10). The microbiota relative abundance was analyzed using UNIFRAC, Principal component analysis (PCA) and Linear Discriminant Analysis Effect Size (LEFSe) between and within groups and also compared to the donor(27, 28). The microbiota analysis was divided in the FMT groups into baseline versus post-antibiotics (day 0 vs. day 5) and using post-FMT samples (day 5 vs days 6 to 20). The Shannon Diversity index was used to define diversity in groups; analysis was performed using paired Wilcoxon tests at different time points within each group and Mann-Whitney between groups. Urine samples were analyzed using NMR spectroscopy using published metabolomic techniques (Supplementary Material)(24).

\section{Statistical Analysis:}

All analyses were performed using the intention-to-treat principle. SAEs were compared between arms at 150 days post-randomization using Fisher exact test. Wilcoxon paired tests were performed within groups at each time point for MELD score, WBC count, AST, ALT, serum albumin and hemoglobin as well as between groups at each time-point using MannWhitney U tests. A similar comparison was performed between and within groups for PHES and EncephalApp-Stroop. A safety power post-hoc calculation gave a power of $83 \%$ for SAE risk between arms, for PHES the power was $84 \%$ and for EncephalApp-Stroop it was $97 \%$. All authors had access to the study data and reviewed and approved the final manuscript.

\section{RESULTS:}

\section{Patients}

Between October 2015 and July 2016, we screened 44 patients. Nine declined and 15 did not meet eligibility. Overall, 20 participants were enrolled in the trial and randomized 1:1 into the FMT or SOC arm (Figure 1A). All participants had similar baseline demographics, laboratory parameters and cirrhosis severity (Table 1).

\section{Adverse Events and Clinical Outcomes}

All subjects randomized to the FMT arm tolerated the pre-treatment antibiotics and the FMT. There were no hospitalizations in the FMT arm for alteration in mental status. Among the FMT arm, two patients were hospitalized within 5 months; one at day 84 post-FMT with acute kidney injury that responded within 24 hours to intravenous hydration and one at day 
115 due to chest pain that was ruled out for an acute cardiac event; both were judged to be unrelated to FMT by the DSMB. Eleven SAEs occurred among 8 participants (80\%) in the SOC arm including 9 events linked to liver-related complications [Six for alteration in mental status, one pneumonia, one for chest pain, one for portal vein thrombus, one for anemia, one for gastroenteritis and two for variceal bleeding (one had HE+variceal bleeding and one had HE+pneumonia)] which was significantly higher than the FMT arm (20\%) ( $\mathrm{p}=0.02$ Table 2 and supplementary table 2). The pneumonia and gastroenteritis occurred at Specifically, the occurrence of HE was also significantly higher in the SOC group (Table 2 and supplementary table 2). Of the 6 episodes, 4 of these required hospitalization for inpatient management. Four episodes were precipitated (one pneumonia, one variceal bleeding, one hyponatremia and one constipation) while the remainder were spontaneous. For those with recurrent HE, since patients were already on maximal HE-related treatment in SOC group and following prescribed diets, no changes apart from those to correct and prevent further precipitating-factors were made. When the safety outcome within 35 days of enrollment was considered, there were two hospitalizations in the SOC group (one patient with variceal bleeding and HE and the other for portal vein thrombus) and none in FMT.

None of the participants in either arm were started on antibiotics unless they developed an infection that was captured above. The pneumonia started at day 89 and was treated with piperacillin/tazobactam and gastroenteritis was deemed to be viral and was self-limited at day 74. Four patients were enrolled in a regular paracentesis program (two in each study arm). The median (IQR) number of paracenteses and associated albumin infusion totals were similar between groups [SOC 6 (2-9) vs. FMT 6 (3-9) paracenteses, albumin SOC $78.3 \pm 24.3$ vs FMT $67.4 \pm 34.1 \mathrm{gm} /$ paracentesis). None of these participants had acute kidney injury or spontaneous bacterial peritonitis noted within seven days of paracentesis as part of the SAEs. All the included patients had evidence of porto-systemic shunting on abdominal imaging and varices on endoscopy and none had transjugular intra-hepatic porto-systemic shunts (TIPS) in place. None of the subjects were lost to follow-up. There were no deaths or transmissible infections noted in either study arm. None of the participants reached a grade of 3 or change in $>2$ of any of their gastrointestinal symptoms according to a standard symptom severity chart (supplementary table 5). None of the participants developed significant changes in a priori safety laboratory parameters (WBC $>12,000$ or $<1000 / \mathrm{mm}^{3}$, MELD $>8$ increase, AST or ALT $>10$ times ULN) which occurred outside the SAEs previously described. The caloric intake and protein intake remained similar within and between groups throughout the trial (Supplementary table 6).

There was no significant change in the MELD scores throughout the trial in the SOC arm. In the FMT arm, use of antibiotics worsened the MELD score (INR and bilirubin were similarly affected; creatinine was stable); however, FMT recovered the MELD score to the baseline value (Table 2, supplementary tables 3 and 4). No changes in WBC count, AST, ALT, albumin or hemoglobin were seen in either arm (Table 2, supplementary table 3). The use of lactulose and rifaximin remained consistent throughout the trial regardless of subject assignment. 


\section{Cognitive Outcomes}

There was a significant improvement in PHES total score $(\mathrm{p}=0.003)$ and EncephalApp Stroop ( $\mathrm{p}=0.01)$ in the FMT group compared to baseline but none in pre- and post-values among the SOC arm (PHES p=0.98, EncephalApp p=0.26, Table 2, Figure 2).

\section{Microbiome and Metabolomic Outcomes}

At baseline, there was similar microbiota composition on weighted UNIFRAC $(\mathrm{p}=0.3)$ and Shannon diversity index ( $\mathrm{p}=0.1$, Mann-Whitney test) between FMT and SOC groups. Postantibiotics, a significant reduction in diversity (Figure 3C) occurred in the FMT arm, which increased post-FMT and returned to the pre-FMT values. No change in SOC diversity indices was observed. In the SOC arm, no significant changes in microbiota composition or diversity were seen throughout the trial (all p>0.05, Figure 3A/C, S3A-E), whereas the FMT arm demonstrated significant compositional changes after antibiotics on UNIFRAC (pvalues $0.01-<1.0 \mathrm{e}-02)$, on PCA, loss of diversity and reduced autochthonous taxa and increased Proteobacteria relative abundance (Figures 3B/C, S2A, S3F/G). A relative increase in beneficial taxa (Lactobacillaceae, Bifidobacteriaceae) was seen post-FMT (Figures $\mathrm{S} 2 \mathrm{~B} / \mathrm{C}, \mathrm{S} 3 \mathrm{H}-\mathrm{J}$ ) compared to post-antibiotics and to the SOC group (Figures S2D/E). Compared to the donor, a significant reduction in relative abundance of Bacteroidetes and expansion of Firmicutes occurred post-antibiotics which increased after FMT. However Proteobacteria, which were low in the donor remained similar across participants apart from the two FMT patients who was subsequently hospitalized (Figure S4A), while the same patients had a persistently lower Lachnospiraceaeae and Ruminococcaceae, which were the highest in the donor (Figure S4B). Overall, patients in the FMT arm had an increase in relative abundance of Lachnospiraceaeae and Ruminococcaceae after FMT compared to their post-antibiotic microbiome, and patients were enriched with Ruminococcaceae postFMT compared to baseline (Figure S4B).

Urinary metabolomics showed that the overall metabolic profile in the SOC arm remained similar over time (Figure S5A). Within the FMT group, metabolites were altered by antibiotics [reduced phenylacetylglutamine ( $\mathrm{PAG}, \mathrm{p}=0.017)$, hippurate $(\mathrm{p}=0.04)$ and formate $(\mathrm{p}=0.007)]$, but returned to baseline post-FMT (Figure S5B, Supplementary table 7). When SOC and FMT groups were compared, antibiotics decreased hippurate, formate and PAG in FMT participants, which largely returned to baseline post-FMT.

\section{DISCUSSION}

Recurrent HE is the leading cause of readmissions in cirrhotic patients despite SOC and may lead to irreversible neuro-cognitive injury(2). HE patients have disrupted gut microbiota, partly driven by frequent antibiotic use, which results in further HE recurrence (6). FMT is a promising therapy to restore a healthy microbiota; however, its safety profile among vulnerable HE patients that are immunosuppressed and at risk of bacteremia due to bacterial translocation is unknown.

In this pilot, open-label randomized clinical trial - to our knowledge, the first FMT trial in HE - we deploy a novel approach by rationally-selecting a stool donor based on cross- 
sectional HE microbiome data. Overall, the primary outcome was met with no FMTassociated SAE, including no bacterial infections. Even more striking was the reduction in hospitalizations due to liver-related, especially portal hypertensive complications, in the FMT arm recognizing a high rate of SOC hospitalizations is expected among those with end-stage liver disease(2). These findings were observed despite adherence to all medications, including lactulose and rifaximin, which was reinforced and inquired upon during each study visit, and both arms balanced for all clinically relevant factors at baseline.

Broad-spectrum pre-treatment antibiotics were used prior to FMT to decrease the host bacterial burden and lower microbial diversity to enable a colonic environment receptive to colonization from a donor's microbiota. $(25,29,30)$ This approach has been demonstrated in FMT for ulcerative colitis, where patients have a low diversity secondary to antibiotics, and clinically improve post-FMT with a corresponding increase in diversity(31). As anticipated, there was a significant disruption of the hosts' microbial diversity with antibiotic pretreatment. However, antibiotics further reduced the already depleted autochthonous taxa(10) beyond HE patients' already dysbiotic baseline (20, 24). The increase in MELD score transiently during antibiotic therapy is interesting and unrelated to direct drug toxicity. Depletion of gut microbial diversity, including autochthonous taxa with changes in Vitamin $\mathrm{K}$ as well as other alterations of the gut-liver axis could be contributory.

Accordingly, the choice to use a single donor enriched in these autochthonous taxa may have helped microbial restoration, including restoring the diversity and enhancement of beneficial taxa such as Ruminococcaceae as well as increasing Bifidobacteriaceae and

Lactobacillaceae. This increase in beneficial taxa could have contributed to the overall improvement in cognitive function and potentially prevention of liver-related hospitalizations(32). Strengthening of intestinal barrier by SCFA production and reducing the relative abundance of potentially pathogenic taxa could be contributory to this $(11,12$, 33-35). These taxa are indicative of a healthy microbiota and their depletion is found in several other diseases such as non-alcoholic fatty liver and $C$.difficile $(36,37)$. We believe these results may be applicable to other studies in $\mathrm{HE}$ given that the sequences sent to map the ideal donor were different from the patients who were ultimately recruited for FMT. Therefore the success of the trial in patients whose stool had not been evaluated a priori could increase the generalizability of these results in other HE patients and centers. These results extend prior studies in which probiotic supplementation with similar taxa improves dysbiosis and reduces hospitalizations in cirrhosis(32, 38-40). These results are a first step for other centers to evaluate strategies that are gut-based which can potentially improve outcomes even after currently available maximal gut-based HE therapy. However, further larger trials are needed. While FMT studies in $C$. difficile have not definitively linked clinical outcome to "donor engraftment", our use of the rationally-derived donor enriched in beneficial taxa likely led to their increase in FMT patients and these findings are not simply related to post-antibiotic recovery.

The PHES and EncephalApp-Stroop are validated HE-related cognitive function tests and performance on these is associated with further HE development, hospitalizations and death $(1,41)$. Independent improvement in these tests in the FMT group is promising and is the first randomized trial data to suggest a clinically meaningful impact of the FMT on the 
gut-liver-brain axis(1,23). Indeed, a participant randomized to the FMT arm whose cognitive performance worsened had a high Proteobacteria relative abundance at baseline with the lowest response to the FMT and was hospitalized. While there were not enough FMT patients with poor outcomes, we speculate that baseline Proteobacteria relative abundance could predict potential microbiota colonization and this requires further validation.

Intriguingly from a mechanism perspective, the MELD score worsened after antibiotic pretreatment due to an increase all three components. This, paralleling the microbiota, was restored after FMT, and was not accompanied by AST/ALT changes. These findings point towards a mechanism unrelated to drug-induced liver injury and could be related to autochthonous taxa depletion, which may perpetuate liver injury in models of antibiotic use(42). Thus, either an infection or subsequent antibiotics may perpetuate liver injury in cirrhosis. Beyond microbiota changes, there were changes in functionality inferred by alterations in urinary mammalian-microbial co-metabolite levels, including formate, hippurate and PAG(43). Previous studies have implicated an increase in formate to higher Proteobacteria taxa, hippurate to Firmicutes taxa and PAG levels to bacterial degradation of phenylalanine(44). The urinary metabolic profile returned towards baseline post-antibiotic treatment and FMT, which supports the interpretation from the microbiome data that antibiotic-associated disruption of the gut-liver axis can be restored by FMT and may prevent further dysbiosis-associated liver injury.

Given the frequency of antibiotic use, the rate of antibiotic-resistant bacterial (ARB) infections in decompensated cirrhotic patients is significant $(4,5)$. Accordingly, the use of antibiotic pre-treatment was approached with caution and an antibiotic-only group or placebo group was not included given the primary safety aim and the objective endpoints of the study. However, a double-therapy approach with FMT was selected given preliminary evidence suggesting the protective ability of FMT to treat multiple ARBs in non-cirrhotic subjects $(45,46)$. Although we did not specifically assess for ARB colonization, no ARB or antibiotic-sensitive infections occurred in the FMT arm.

This study has several limitations. First, combined pre-treatment antibiotics and FMT makes it difficult to discern the precise role of FMT alone. However, given the potential for ARB infections, the use of antibiotics alone as a control arm was deemed unethical. Additionally, pretreatment antibiotics were felt necessary to facilitate colonization of donor microbiota, although there is a paucity of robust clinical evidence on the degree it contributes to efficacy. Future studies, using FMT alone may clarify this question. Second, the control arm was SOC not placebo antibiotics and sham FMT or autologous FMT. However, the primary and key secondary outcomes are objective, assessed by the independent DSMB, and unlikely driven by a placebo effect. Third, the dose of fecal material, dosing frequency, ideal donor and delivery modality are unknown since these can affect FMT efficacy in other diseases(47, 48). Specifically, given the selected donor approach, the results may not be generalized to FMT from different donors and administration routes(49). Fourth, the precise mechanism of FMT still requires further elucidation but we speculate the single donor enriched in the beneficial, autochthonous taxa could be key and the expansion of potentially probiotic taxa. Fifth, given the small sample size, further larger studies are needed to confirm this outcome 
in larger sample sizes and other settings, including among women and in those with MELD scores higher than 17 to refine the treatment approach. Lastly, we only performed cognitive and microbiota analyses till 20 days. A longer period of cognitive and microbiota follow-up as well as patient-reported outcomes beyond AEs would have yielded greater insight into these changes post-FMT. However, regardless of the size the treatment effect is noteworthy in this population with limited therapeutic options.

We conclude that in this small randomized trial, FMT from a rationally selected donor was safe, associated with lower hospitalizations and improved cognitive tests among cirrhotic patients with recurrent HE.

\section{Supplementary Material}

Refer to Web version on PubMed Central for supplementary material.

\section{Acknowledgements:}

We thank Dr Abil Aliev, Department of Chemistry, University College London, London, for use of the NMR spectrometer. SDT-R acknowledges the UK NIHR Biomedical Facility at Imperial College London for infrastructure support. ZK and MS thank the Neil \& Anna Rasmussen Foundation and Anna-Maria and Stephen Kellen Foundation for support of OpenBiome, a non-profit universal stool bank. The investigators acknowledge Dr Douglas Heuman, HoChong Gilles and Dr Michael Climo, who constituted the Data Safety Monitoring Board and Brent Gregory, PharmD who supervised the McGuire VAMC Investigational Pharmacy and Dr Narayan Dharel for help with recruitment.

Financial support: The trial was funded partly by McGuire Research Institute and CX10076 VA Merit Review awarded to JSB. None of the funders had any role in the design and conduct of the study; collection, management, analysis, and interpretation of the data; preparation, review, or approval of the manuscript; and decision to submit the manuscript for publication

\section{Abbreviations:}

$\begin{array}{ll}\text { HE } & \text { hepatic encephalopathy } \\ \text { SOC } & \text { standard of care } \\ \text { MELD } & \text { model for end-stage liver disease } \\ \text { FMT } & \text { fecal microbiota transplantation } \\ \text { SAE } & \text { serious adverse events } \\ \text { AE } & \text { adverse events } \\ \text { DSMB } & \text { data safety monitoring board } \\ \text { TIPS } & \text { transjugular intra-hepatic porto-systemic shunts } \\ \text { PHES } & \text { psychometric hepatic encephalopathy score } \\ \text { ULN } & \text { upper limit of normal } \\ \text { NMR } & \text { nuclear magnetic resonance } \\ \text { PAG } & \text { phenylacetylglutamine }\end{array}$




$\begin{array}{ll}\text { PCA } & \text { Principal component analysis (PCA) } \\ \text { LEFSe } & \text { Linear Discriminant Analysis Effect Size } \\ \text { SCFA } & \text { short-chain fatty acid }\end{array}$

\section{References:}

1. Vilstrup H, Amodio P, Bajaj J, Cordoba J, Ferenci P, Mullen KD, Weissenborn K, et al. Hepatic encephalopathy in chronic liver disease: 2014 Practice Guideline by the American Association for the Study of Liver Diseases and the European Association for the Study of the Liver. Hepatology 2014;60:715-735. [PubMed: 25042402]

2. Bajaj JS, Reddy KR, Tandon P, Wong F, Kamath PS, Garcia-Tsao G, Maliakkal B, et al. The 3month readmission rate remains unacceptably high in a large North American cohort of patients with cirrhosis. Hepatology 2016;64:200-208. [PubMed: 26690389]

3. Stepanova M, Mishra A, Venkatesan C, Younossi ZM. In-hospital mortality and economic burden associated with hepatic encephalopathy in the United States from 2005 to 2009. Clin Gastroenterol Hepatol 2012;10:1034-1041 e1031. [PubMed: 22642955]

4. Fernandez J, Tandon P, Mensa J, Garcia-Tsao G. Antibiotic prophylaxis in cirrhosis: Good and bad. Hepatology 2016;63:2019-2031. [PubMed: 26528864]

5. Bajaj JS, O'Leary JG, Reddy KR, Wong F, Olson JC, Subramanian RM, Brown G, et al. Second infections independently increase mortality in hospitalized patients with cirrhosis: the North American consortium for the study of end-stage liver disease (NACSELD) experience. Hepatology 2012;56:2328-2335. [PubMed: 22806618]

6. Bajaj JS, Schubert CM, Heuman DM, Wade JB, Gibson DP, Topaz A, Saeian K, et al. Persistence of cognitive impairment after resolution of overt hepatic encephalopathy. Gastroenterology 2010;138:2332-2340. [PubMed: 20178797]

7. Bajaj JS, Heuman DM, Sanyal AJ, Hylemon PB, Sterling RK, Stravitz RT, Fuchs M, et al. Modulation of the metabiome by rifaximin in patients with cirrhosis and minimal hepatic encephalopathy. PLoS One 2013;8:e60042. [PubMed: 23565181]

8. Bajaj JS, Gillevet PM, Patel NR, Ahluwalia V, Ridlon JM, Kettenmann B, Schubert CM, et al. A longitudinal systems biology analysis of lactulose withdrawal in hepatic encephalopathy. Metab Brain Dis 2012;27:205-215. [PubMed: 22527995]

9. Chen Y, Yang F, Lu H, Wang B, Chen Y, Lei D, Wang Y, et al. Characterization of fecal microbial communities in patients with liver cirrhosis. Hepatology 2011;54:562-572. [PubMed: 21574172]

10. Bajaj JS, Heuman DM, Hylemon PB, Sanyal AJ, White MB, Monteith P, Noble NA, et al. Altered profile of human gut microbiome is associated with cirrhosis and its complications. J Hepatol 2014;60:940-947. [PubMed: 24374295]

11. Pryde SE, Duncan SH, Hold GL, Stewart CS, Flint HJ. The microbiology of butyrate formation in the human colon. FEMS Microbiol Lett 2002;217:133-139. [PubMed: 12480096]

12. Barcenilla A, Pryde SE, Martin JC, Duncan SH, Stewart CS, Henderson C, Flint HJ. Phylogenetic relationships of butyrate-producing bacteria from the human gut. Appl Environ Microbiol 2000;66:1654-1661. [PubMed: 10742256]

13. Reichardt N, Duncan SH, Young P, Belenguer A, McWilliam Leitch C, Scott KP, Flint HJ, et al. Phylogenetic distribution of three pathways for propionate production within the human gut microbiota. ISME J 2014;8:1323-1335. [PubMed: 24553467]

14. Bajaj JS, Ridlon JM, Hylemon PB, Thacker LR, Heuman DM, Smith S, Sikaroodi M, et al. Linkage of gut microbiome with cognition in hepatic encephalopathy. Am J Physiol Gastrointest Liver Physiol 2012;302:G168-175. [PubMed: 21940902]

15. Ahluwalia V, Wade JB, Moeller FG, White MB, Unser AB, Gavis EA, Sterling RK, et al. The etiology of cirrhosis is a strong determinant of brain reserve: A multimodal magnetic resonance imaging study. Liver Transpl 2015;21:1123-1132. [PubMed: 25939692]

16. Smith MB, Kelly C, Alm EJ. Policy: How to regulate faecal transplants. Nature 2014;506:290-291. [PubMed: 24558658] 
17. van Nood E, Vrieze A, Nieuwdorp M, Fuentes S, Zoetendal EG, de Vos WM, Visser CE, et al. Duodenal infusion of donor feces for recurrent Clostridium difficile. N Engl J Med 2013;368:407415. [PubMed: 23323867]

18. Moayyedi P, Surette MG, Kim PT, Libertucci J, Wolfe M, Onischi C, Armstrong D, et al. Fecal Microbiota Transplantation Induces Remission in Patients With Active Ulcerative Colitis in a Randomized Controlled Trial. Gastroenterology 2015;149:102-109 e106. [PubMed: 25857665]

19. Kelly CR, Khoruts A, Staley C, Sadowsky MJ, Abd M, Alani M, Bakow B, et al. Effect of Fecal Microbiota Transplantation on Recurrence in Multiply Recurrent Clostridium difficile Infection: A Randomized Trial. Ann Intern Med 2016;165:609-616. [PubMed: 27547925]

20. Bajaj JS, Betrapally NS, Gillevet PM. Decompensated cirrhosis and microbiome interpretation. Nature 2015;525:E1-2. [PubMed: 26381988]

21. Kao D, Roach B, Park H, Hotte N, Madsen K, Bain V, Tandon P. Fecal microbiota transplantation in the management of hepatic encephalopathy. Hepatology 2016;63:339-340. [PubMed: 26264779]

22. Malinchoc M, Kamath PS, Gordon FD, Peine CJ, Rank J, ter Borg PC. A model to predict poor survival in patients undergoing transjugular intrahepatic portosystemic shunts. Hepatology 2000;31:864-871. [PubMed: 10733541]

23. Allampati S, Duarte-Rojo A, Thacker LR, Patidar KR, White MB, Klair JS, John B, et al. Diagnosis of Minimal Hepatic Encephalopathy Using Stroop EncephalApp: A Multicenter USBased, Norm-Based Study. Am J Gastroenterol 2016;111:78-86. [PubMed: 26644276]

24. Bajaj JS, Cox IJ, Betrapally NS, Heuman DM, Schubert ML, Ratneswaran M, Hylemon PB, et al. Systems Biology Analysis of Omeprazole Therapy in Cirrhosis Demonstrates Significant Shifts in Gut Microbiota Composition and Function. Am J Physiol Gastrointest Liver Physiol 2014.

25. Dethlefsen L, Huse S, Sogin ML, Relman DA. The pervasive effects of an antibiotic on the human gut microbiota, as revealed by deep 16S rRNA sequencing. PLoS Biol 2008;6:e280. [PubMed: 19018661]

26. Lee CH, Steiner T, Petrof EO, Smieja M, Roscoe D, Nematallah A, Weese JS, et al. Frozen vs Fresh Fecal Microbiota Transplantation and Clinical Resolution of Diarrhea in Patients With Recurrent Clostridium difficile Infection: A Randomized Clinical Trial. JAMA 2016;315:142-149. [PubMed: 26757463]

27. Segata N, Izard J, Waldron L, Gevers D, Miropolsky L, Garrett WS, Huttenhower C. Metagenomic biomarker discovery and explanation. Genome Biol 2011;12:R60. [PubMed: 21702898]

28. http://qiime.org/tutorials/tutorial.html. In.

29. Lankelma JM, Belzer C, Hoogendijk AJ, de Vos AF, de Vos WM, van der Poll T, Wiersinga WJ. Antibiotic-Induced Gut Microbiota Disruption Decreases TNF-alpha Release by Mononuclear Cells in Healthy Adults. Clin Transl Gastroenterol 2016;7:e186. [PubMed: 27489950]

30. van Nood E, Dijkgraaf MG, Keller JJ. Duodenal infusion of feces for recurrent Clostridium difficile. N Engl J Med 2013;368:2145.

31. Ishikawa D, Sasaki T, Osada T, Kuwahara-Arai K, Haga K, Shibuya T, Hiramatsu K, et al. Changes in Intestinal Microbiota Following Combination Therapy with Fecal Microbial Transplantation and Antibiotics for Ulcerative Colitis. Inflamm Bowel Dis 2017;23:116-125. [PubMed: 27893543]

32. Dhiman RK, Rana B, Agrawal S, Garg A, Chopra M, Thumburu KK, Khattri A, et al. Probiotic VSL\#3 reduces liver disease severity and hospitalization in patients with cirrhosis: a randomized, controlled trial. Gastroenterology 2014;147:1327-1337 e1323. [PubMed: 25450083]

33. Jones EA. Ammonia, the GABA neurotransmitter system, and hepatic encephalopathy. Metab Brain Dis 2002;17:275-281. [PubMed: 12602504]

34. Bercik P, Collins SM, Verdu EF. Microbes and the gut-brain axis. Neurogastroenterol Motil 2012;24:405-413. [PubMed: 22404222]

35. Wong JM, de Souza R, Kendall CW, Emam A, Jenkins DJ. Colonic health: fermentation and short chain fatty acids. J Clin Gastroenterol 2006;40:235-243. [PubMed: 16633129]

36. Antharam VC, Li EC, Ishmael A, Sharma A, Mai V, Rand KH, Wang GP. Intestinal dysbiosis and depletion of butyrogenic bacteria in Clostridium difficile infection and nosocomial diarrhea. J Clin Microbiol 2013;51:2884-2892. [PubMed: 23804381] 
37. Wang B, Jiang X, Cao M, Ge J, Bao Q, Tang L, Chen Y, et al. Altered Fecal Microbiota Correlates with Liver Biochemistry in Nonobese Patients with Non-alcoholic Fatty Liver Disease. Sci Rep 2016;6:32002. [PubMed: 27550547]

38. Lunia MK, Sharma BC, Sharma P, Sachdeva S, Srivastava S. Probiotics prevent hepatic encephalopathy in patients with cirrhosis: a randomized controlled trial. Clin Gastroenterol Hepatol 2014;12:1003-1008 e1001. [PubMed: 24246768]

39. Bajaj JS, Heuman DM, Hylemon PB, Sanyal AJ, Puri P, Sterling RK, Luketic V, et al. Randomised clinical trial: Lactobacillus GG modulates gut microbiome, metabolome and endotoxemia in patients with cirrhosis. Aliment Pharmacol Ther 2014;39:1113-1125. [PubMed: 24628464]

40. Bajaj JS, Saeian K, Christensen KM, Hafeezullah M, Varma RR, Franco J, Pleuss JA, et al. Probiotic yogurt for the treatment of minimal hepatic encephalopathy. Am J Gastroenterol 2008;103:1707-1715. [PubMed: 18691193]

41. Allampati S, Mullen KD. Does overt hepatic encephalopathy cause persistent cognitive defects even after successful liver transplantation? Liver Transpl 2014;20:874-875. [PubMed: 24961815]

42. Lewis JH. The Art and Science of Diagnosing and Managing Drug-induced Liver Injury in 2015 and Beyond. Clin Gastroenterol Hepatol 2015;13:2173-2189 e2178. [PubMed: 26116527]

43. Yap IK, Li JV, Saric J, Martin FP, Davies H, Wang Y, Wilson ID, et al. Metabonomic and microbiological analysis of the dynamic effect of vancomycin-induced gut microbiota modification in the mouse. J Proteome Res 2008;7:3718-3728. [PubMed: 18698804]

44. Williams HR, Cox IJ, Walker DG, North BV, Patel VM, Marshall SE, Jewell DP, et al. Characterization of inflammatory bowel disease with urinary metabolic profiling. Am J Gastroenterol 2009;104:1435-1444. [PubMed: 19491857]

45. Singh R, van Nood E, Nieuwdorp M, van Dam B, ten Berge IJ, Geerlings SE, Bemelman FJ. Donor feces infusion for eradication of Extended Spectrum beta-Lactamase producing Escherichia coli in a patient with end stage renal disease. Clin Microbiol Infect 2014;20:O977-978. [PubMed: 24845223]

46. Crum-Cianflone NF, Sullivan E, Ballon-Landa G. Fecal microbiota transplantation and successful resolution of multidrug-resistant-organism colonization. J Clin Microbiol 2015;53:1986-1989. [PubMed: 25878340]

47. Drekonja D, Reich J, Gezahegn S, Greer N, Shaukat A, MacDonald R, Rutks I, et al. Fecal Microbiota Transplantation for Clostridium difficile Infection: A Systematic Review. Ann Intern Med 2015;162:630-638. [PubMed: 25938992]

48. Kassam Z, Lee CH, Yuan Y, Hunt RH. Navigating long-term safety in fecal microbiota transplantation. Am J Gastroenterol 2013;108:1538.

49. Philips CA, Pande A, Shasthry SM, Jamwal KD, Khillan V, Chandel SS, Kumar G, et al. Healthy Donor Fecal Microbiota Transplantation in Steroid Ineligible Severe Alcoholic Hepatitis - A Pilot Study. Clin Gastroenterol Hepatol 2016. 


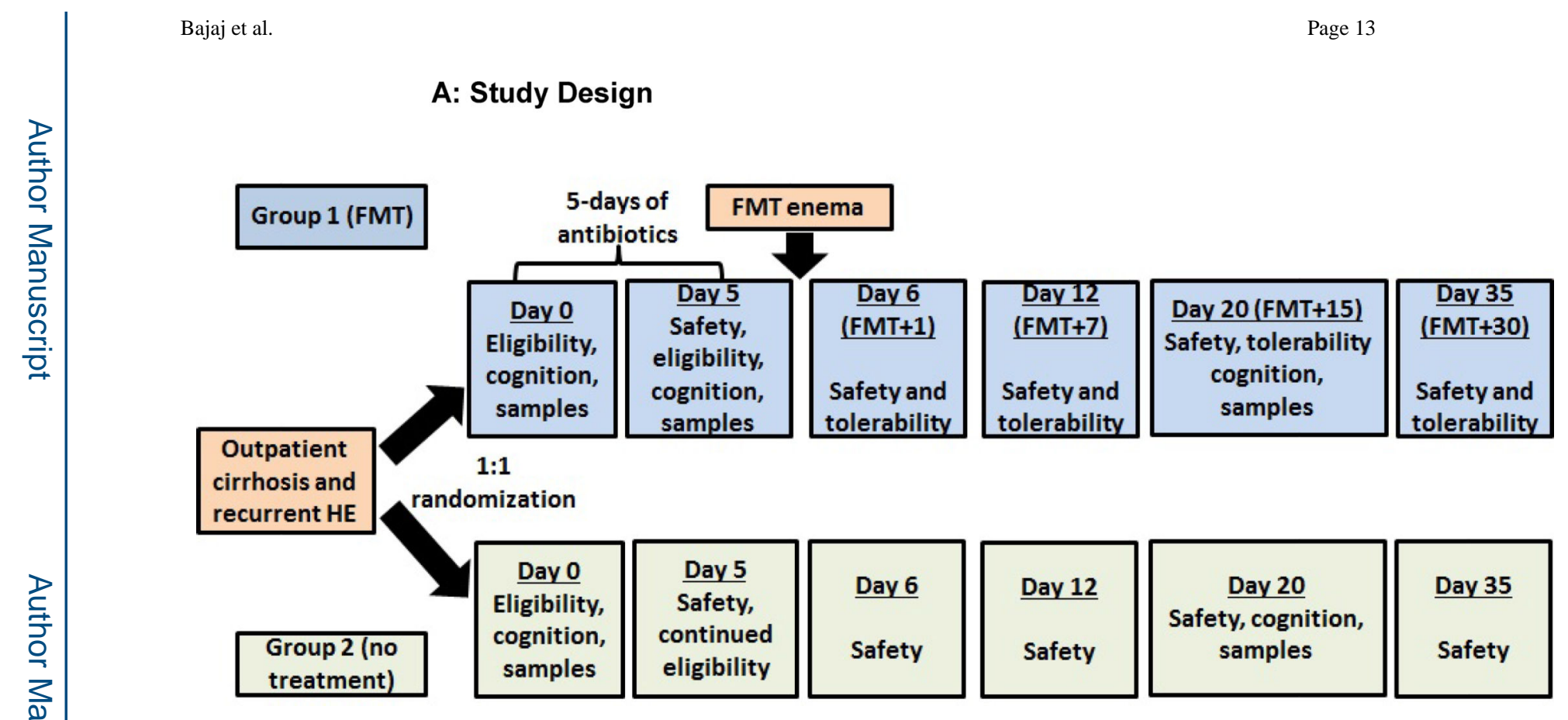




\section{B: Enrollment (CONSORT Flowchart)}

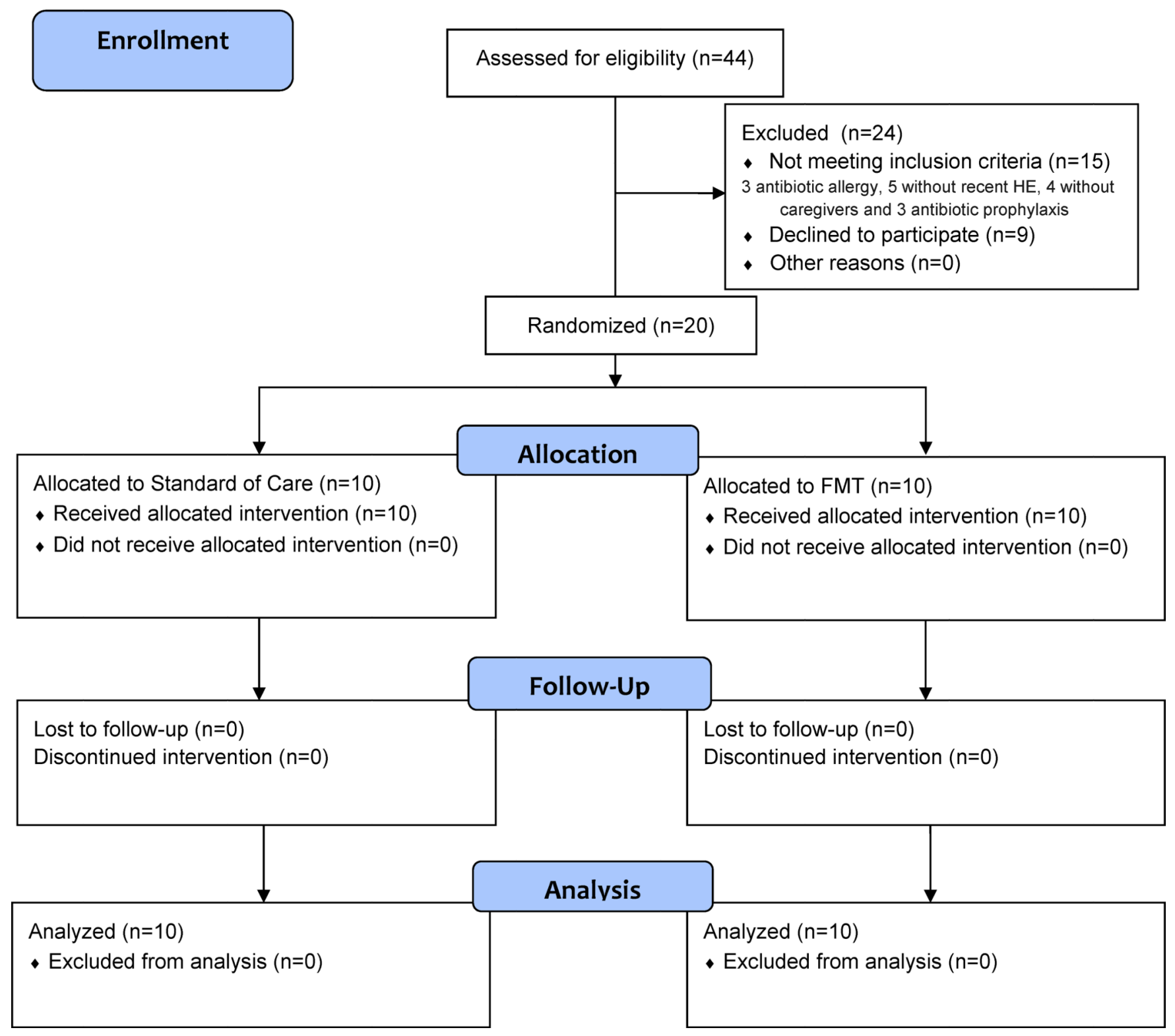

Figure 1:

Patient flow and study design;

Figure 1A: Flow chart of study design; patients were followed for 150 days after enrollment. At day 5 after randomization (post-antibiotics in the FMT arm), blood, urine and stool was collected from all participants and adverse events (AE), new medications and diagnoses as well as adherence to the original diet was documented. All participants were seen at day 6 after randomization (day 1 post-FMT in the FMT arm) where a solicited/unsolicited AE assessment, safety laboratories (MELD score, liver function tests, $\mathrm{CBC}$ ), urine and stool was 
collected. Similarly, at days 12 and 20 post-randomization, we performed safety laboratories, collected blood, urine and stool, and evaluated AEs. At day 20, both cognitive tests were re-administered. At day 35, we collected safety laboratories and evaluated AEs. At day 150, SAE assessment was conducted.

Figure 1B: CONSORT Flow chart showing that there were no dropouts and all subjects were followed throughout the study 


\section{Cognitive Changes in FMT vs SOC group}
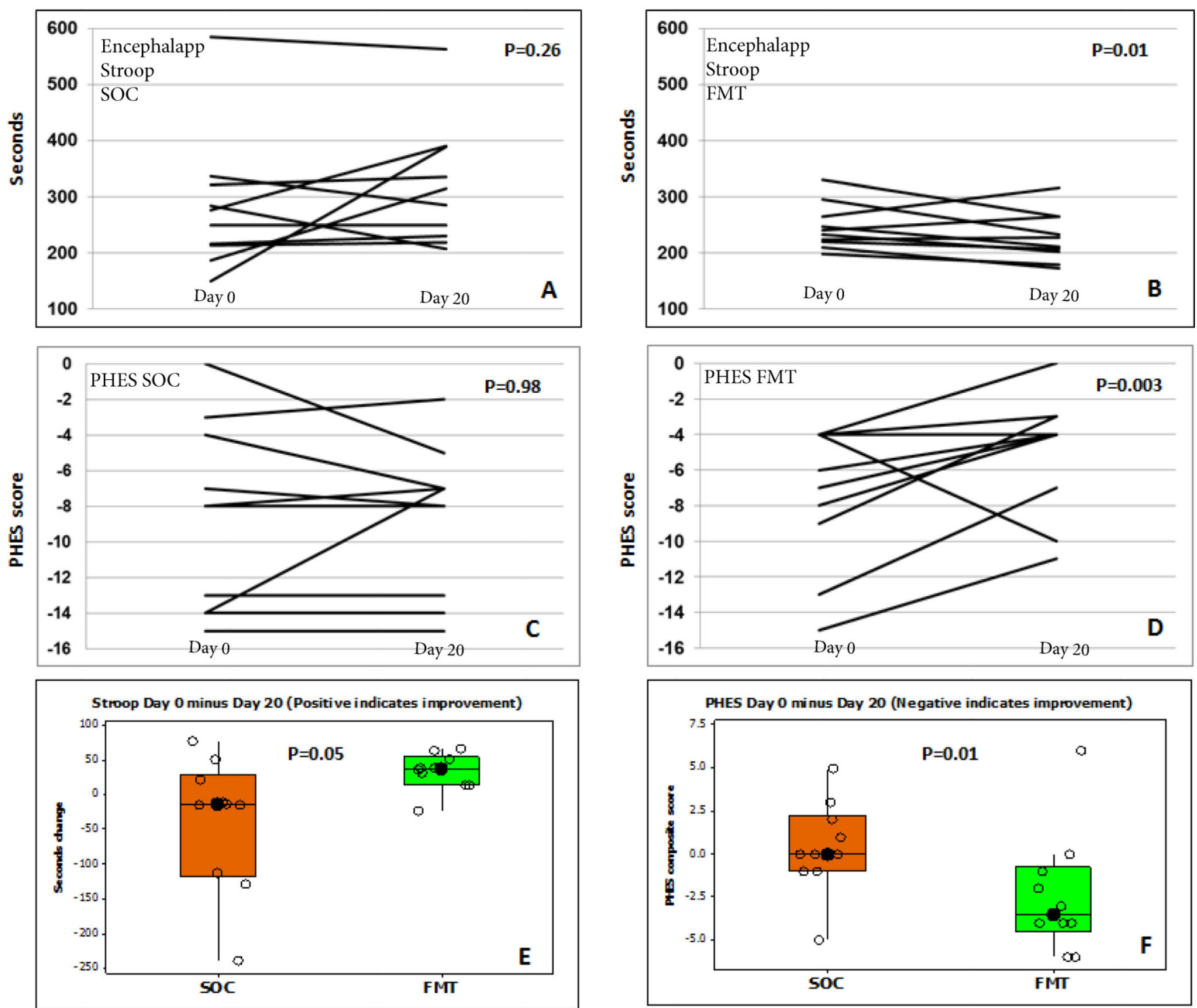

Figure 2:

Cognitive function changes; FMT: fecal microbiota transplant, SOC: standard of care, PHES: psychometric hepatic encephalopathy score

Figure 2A: EncephalApp Stroop in SOC group shows no significant change in OffTime +OnTime in seconds compared to baseline. The first data point is day 0 and the second data point is day 20. This was not significant even when the outlier subject values were removed Figure 2B: EncephalApp Stroop in FMT group showed significant improvement (reduction) in OffTime+OnTime in seconds compared to baseline. The first data point is day 0 and the second data point is day 20 (day 15 post-FMT) 
Figure 2C: PHES score in the SOC group did not show any change in the SOC group. The first data point is day 0 and the second data point is day 20 . Y axis is the sum of standard deviations of PHES scores compared to healthy controls

Figure 2D: PHES score in the FMT group significantly improved (decreased) compared to baseline. The first data point is day 0 and the second data point is day 20 (day 15 post-FMT). Figure 2E: Delta in EncephalApp Stroop in baseline minus day 20 (negative values indicate improvement) shows that there was a significant improvement in FMT compared to SOC group. Data are shown as median and $95 \% \mathrm{CI}$ intervals.

Figure 2F: Delta in PHES in baseline minus day 20 showed a significant improvement (positive values indicate improvement) in FMT compared to SOC group. Data are shown as median and $95 \% \mathrm{CI}$ intervals. 


\section{Microbial changes}

A: Principal Component Analysis within the FMT group

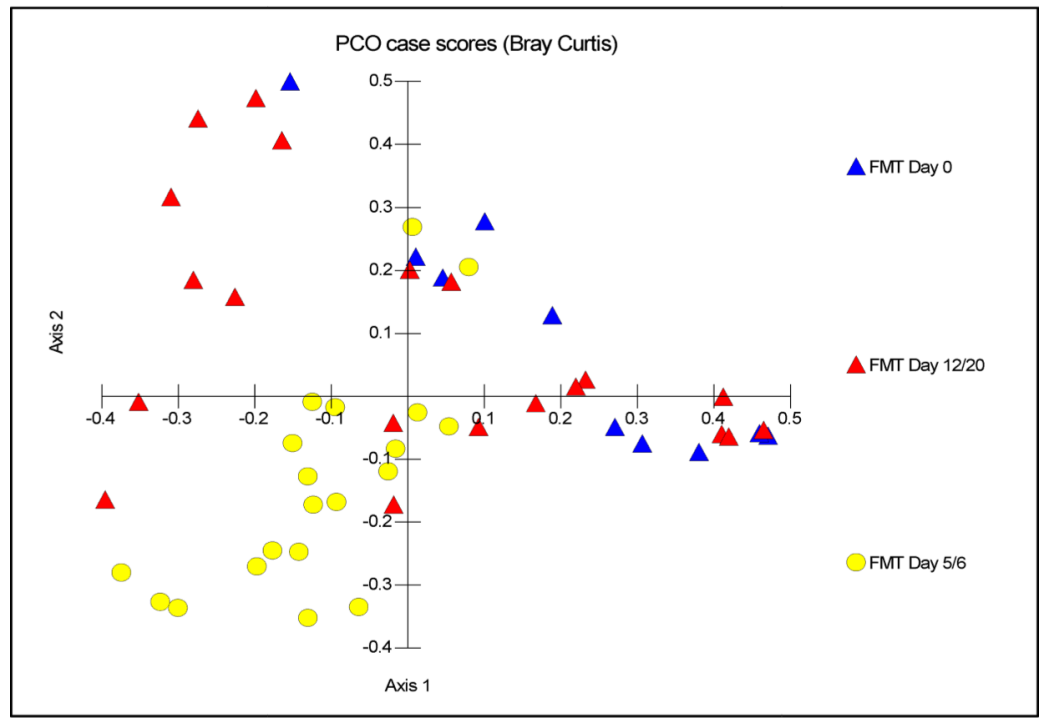

B: Principal Component Analysis within the SOC group

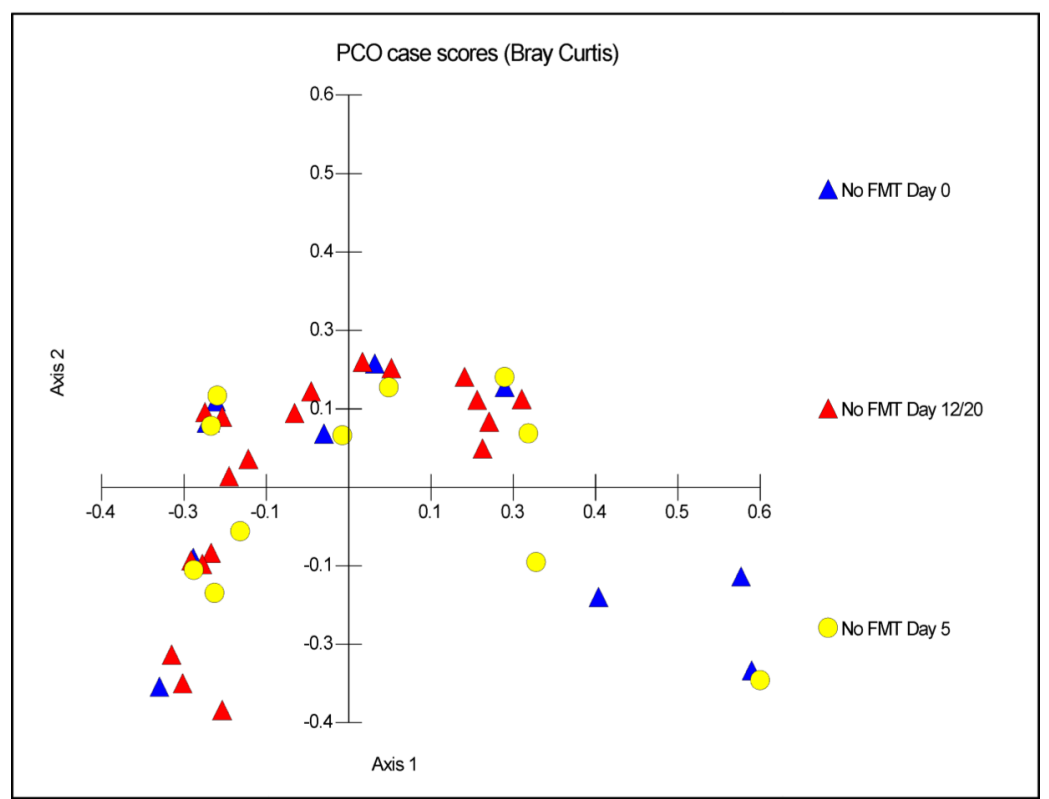

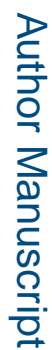

Hepatology. Author manuscript; available in PMC 2018 December 01. 


\section{C: Microbial Diversity between and within FMT and SOC groups}

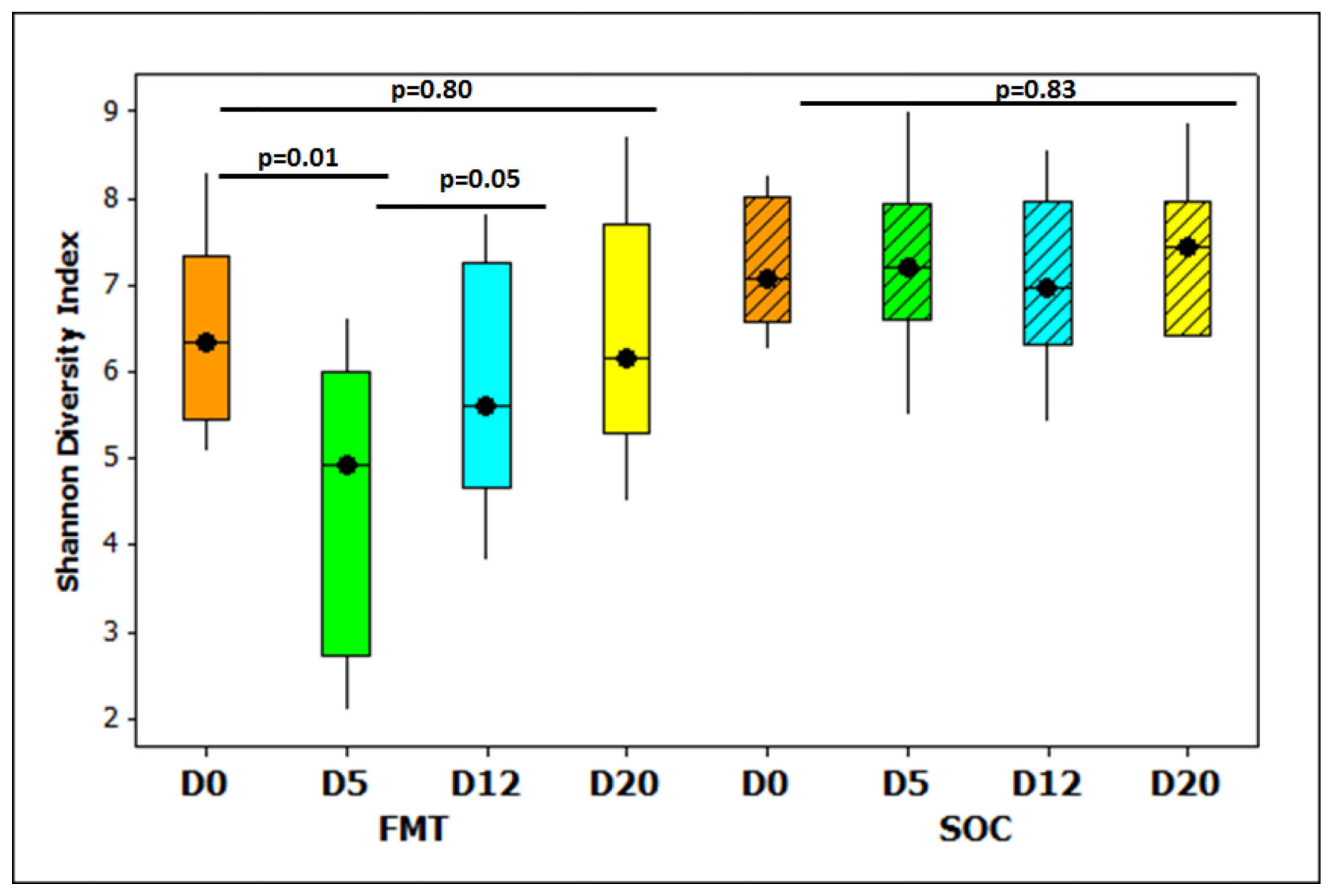

Figure 3:

Microbial changes throughout the trial, FMT: fecal microbiota transplant, SOC: standard of care, PCA: Principal component analysis,

Figure 3A: PCA of gut microbiota in patients in the FMT group, Blue triangles: baseline values, Yellow circles: day 5 and 6 and Red triangles: days 12 and 20. There was relative clustering of the stool microbiota at days 5 and 6 away from day 0 , while at day 12/20, these reached close to the day 0 values.

Figure 3B: PCA of gut microbiota in patients in the SOC group; blue triangles: baseline, yellow circles: day 5 and red triangles: days 12 and 20. There was no specific clustering observed at any time point

Figure 3C: Shannon diversity index changes over time; there was a significant reduction in Shannon index (decreased diversity after antibiotics) at day 5, which resolved significantly post-FMT. No changes were seen in the SOC group. D0: day 0, baseline, D5: postantibiotics in FMT group before FMT; no intervention in SOC group, D12: 7 days post-FMT and D20: 15 days post-FMT in FMT group. Boxes in shaded colors indicate SOC group. Data are shown as median and $95 \%$ CI intervals. 
Table 1:

Baseline Demographics and Clinical Characteristics

\begin{tabular}{|l|c|c|c|}
\hline Mean \pm standard deviation unless stated otherwise & $\begin{array}{c}\text { SOC arm } \\
(\mathbf{n}=10)\end{array}$ & $\begin{array}{c}\text { FMT arm } \\
(\mathbf{n}=10)\end{array}$ & P value \\
\hline Age (years) & $62.9 \pm 9.8$ & $64.5 \pm 5.1$ & 0.65 \\
\hline Gender (Men/Women) & $10 / 0$ & $10 / 0$ & 1.0 \\
\hline Race (Caucasian, African-American, Hispanic, others) & $6 / 3 / 1 / 0$ & $7 / 2 / 1 / 0$ & 0.9 \\
\hline Diabetes (\%) & $40 \%$ & $30 \%$ & 0.63 \\
\hline Etiology (HCV/Alcohol/HCV+Alcohol/ Non-alcoholic fatty liver/others) & $1 / 5 / 2 / 1 / 1$ & $2 / 4 / 2 / 2 / 0$ & 0.46 \\
\hline Prior ascites (\%) & $90 \%$ & $80 \%$ & 0.24 \\
\hline Prior variceal bleeding (\%) & $40 \%$ & $50 \%$ & 0.65 \\
\hline Last HE episode (months prior to enrollment) & $5.9 \pm 3.8$ & $4.5 \pm 3.2$ & 0.39 \\
\hline No. of total HE episodes median (IQR) & $2(2,3)$ & $2(2,3)$ & 0.96 \\
\hline Last Hospitalization (months prior to enrollment) & $5.3 \pm 2.4$ & $7.2 \pm 8.3$ & 0.31 \\
\hline Continued & SOC arm & FMT arm & P value \\
\hline Proton pump inhibitors (\%) & $100 \%$ & $100 \%$ & 1.0 \\
\hline Non-selective beta-blockers (\%) & $50 \%$ & $50 \%$ & 1.0 \\
\hline Hemoglobin (g/dl) & $12.2 \pm 2.1$ & $12.3 \pm 2.0$ & 0.95 \\
\hline Serum WBC count (/mm $\left.{ }^{3}\right)$ & $4.2 \pm 0.9$ & $5.6 \pm 1.6$ & 0.02 \\
\hline MELD score & $13.2 \pm 3.7$ & $12.0 \pm 2.9$ & 0.36 \\
\hline Serum albumin (g/dl) & $3.1 \pm 0.5$ & $3.2 \pm 0.4$ & 0.43 \\
\hline Serum AST & $58.0 \pm 37.4$ & $48.6 \pm 17.3$ & 0.48 \\
\hline Serum ALT & $44.8 \pm 25.2$ & $41.5 \pm 17.9$ & 0.74 \\
\hline Overall PHES score & $-8.6 \pm 5.3$ & $-7.4 \pm 3.9$ & 0.57 \\
\hline EncephalApp Stroop OffTime+OnTime (seconds) & $282 \pm 122$ & $247 \pm 41$ & 0.40 \\
\hline
\end{tabular}

Apart from a higher baseline WBC count among the FMT arm, all other characteristics were statistically similar between groups. SOC: standard of care, FMT: fecal microbial transplant, HCV: hepatitis C virus, PHES: psychometric hepatic encephalopathy (a lower score indicates worse cognitive performance), on the EncephalApp Stroop a higher time required indicates worse cognitive performance. 
Table 2:

\section{Clinical Outcomes}

\begin{tabular}{|l|c|c|c|}
\hline Mean \pm standard deviation unless mentioned otherwise & $\begin{array}{c}\text { SOC arm } \\
(\mathbf{n}=\mathbf{1 0})\end{array}$ & $\begin{array}{c}\text { FMT arm } \\
(\mathbf{n}=\mathbf{1 0})\end{array}$ & P value \\
\hline Patients with SAEs at day 150 & $8(80 \%)$ & $2(20 \%)$ & 0.02 \\
\hline Total SAEs at day 150* & 11 & 2 & 0.01 \\
\hline Patients with altered mental status by day 150 & 5 & 0 & 0.03 \\
\hline Total HE episodes at day 150* & 6 & 0 & 0.03 \\
\hline Stroop OffTime+OnTime change (day 0 and day 20) positive indicates improvement & $-43.5 \pm 95.7$ & $29.1 \pm 27.9$ & 0.04 \\
\hline PHES score change (day 0 and day 20) negative indicates improvement & $0.0 \pm 3.1$ & $-3.1 \pm 2.1$ & 0.01 \\
\hline MELD score change (day 0 and day 35) & $-0.2 \pm 2.7$ & $0.1 \pm 2.0$ & 0.78 \\
\hline Serum albumin (mg/dl) change (day 0 and day 35) & $0.02 \pm 0.16$ & $0.0 \pm 0.21$ & 0.79 \\
\hline Continued & $\begin{array}{c}\text { SOC arm } \\
(\mathrm{n}=10)\end{array}$ & $\begin{array}{c}\text { FMT arm } \\
(\mathrm{n}=10)\end{array}$ & P value \\
\hline Serum AST (IU) change (day 0 and day 35) & $4.4 \pm 17.2$ & $-0.6 \pm 1.9$ & 0.46 \\
\hline Serum ALT (IU) change (day 0 and day 35) & $1.44 \pm 9.3$ & $-3.8 \pm 6.4$ & 0.18 \\
\hline WBC count (/mm )change (day 0 and day 35) & $0.22 \pm 0.47$ & $0.20 \pm 0.91$ & 0.95 \\
\hline Hemoglobin $(\mathrm{g} / \mathrm{dl})$ change (day 0 and day 35) & $0.4 \pm 0.65$ & $0.0 \pm 1.0$ & 0.29 \\
\hline
\end{tabular}

A significantly higher proportion of participants in the SOC arm experienced serious adverse events and the number of hospitalizations were also the higher in this group. There was also an improvement in cognitive function in the FMT group, while hepatic function and blood count variables changed similarly between groups. SOC: standard of care, FMT: fecal microbial transplant

* Mann-Whitney U test 